\title{
Solution Generation through Hybrid Intelligence and Creativity based on Investment Portfolio
}

\author{
Qinyun Liu ${ }^{\mathrm{a}}$, Hua Zhou ${ }^{\mathrm{b}}$, Hongji Yang ${ }^{\mathrm{c}}$, and William Cheng Chung Chu ${ }^{\mathrm{d}, *}$ \\ ${ }^{a}$ Centre for Creative Computing, Bath Spa University, Bath, BA2 9BN, England \\ ${ }^{b}$ College of Big Data and Intelligence Engineering, Southwest Forestry University, Kunming, 650000, China \\ ${ }^{C}$ Department of Informatics, University of Leicester, Leicester, LE1 7RH, England \\ ${ }^{d}$ Department of Computer Science, Tunghai University, Taichung, 40704, Taiwan, China
}

\begin{abstract}
Artificial Intelligence (AI) has been developed to be robust on computing. Learning can be achieved by connecting to heterogeneous data using AI algorithms, such as the Artificial Neural Network. Knowledge can be learned, and rules in the database can be discovered by machines through heuristic algorithms. However, creativity has not been achieved by computers like the human brain by using AI algorithms individually. This research serves to explore a method to achieve creative solution generation by utilizing a relationship between intelligence and creativity, assuming intelligence is the subset of creativity. Under this relationship, the computing can be fulfilled using AI algorithms. The theories of achieving creativity is the guidance of this method.
\end{abstract}

Keywords: intelligence; creativity; artificial neural network; semantic web; creative computing

(Submitted on April 16, 2018; Revised on May 19, 2018; Accepted on June 16, 2018)

(C) 2018 Totem Publisher, Inc. All rights reserved.

\section{Introduction}

At least five relationships between intelligence and creativity have been proposed, i.e., intelligence being a subset of creativity, creativity being a subset of intelligence, intelligence equalling creativity, intelligence and creativity having a coincident set, and intelligence and creativity being unrelated. To achieve creativity, researching on the relationships between intelligence and creativity is imperative. Each of the above relationships can be used for applications. This research focuses on the relationship where intelligence (I) is a subset of creativity (C). Based on the I $\subset$ C, a solution generation system in an application domain is established.

Many algorithms have been built through developing artificial intelligence, e.g., a heuristic method of neural network and a simulative annealing algorithm. While efficient AI algorithms are being developed, generating creativity is drawing attention of researchers, under the observation that computers are unable to achieve many human activities such as thinking, imagining, and meditation.

However, similar to the birth of AI, artificial creativity could also be achieved using heuristic methods. Considering the cognitive process in human brains, the initial step of learning knowledge is to save it in the neural cells, which is named fluid intelligence [5]. Then, knowledge is possibly utilised by different methods, one of which is a creative method, such as divergent thinking [1], to achieve novel ideas. Scholars proposed three methods to achieve humans' creative ideas through computers: the combinational method, transformational method, and exploratory method [11]. In this process, intelligence information and approaches are implemented under the guidance of creative theory. The relationships between intelligence and creativity is I $\subset$ C.

\footnotetext{
* Corresponding author.

E-mail address: cchu@ thu.edu.tw
} 
To establish a solution generation system, three phases, i.e., query analysis, information expansion, and creative solution generation, are required. Sternberg and Lubart's investment theory, the triarchic theory, and Smith's hierarchy are basic guidance for generating solutions. A natural language process, Artificial Neural Network (Back-propagation neural network and self-organised map neural network), dimension reduction algorithm (principle components analysis), and association rules learning (Apriori) are used to analyse data. Semantic Web is used to manipulate information to simulate divergent thinking. To describe the SGIC system more explicitly, an investment portfolio case study will be presented. This paper has five sections: introduction, related work, main approach, experiment, and conclusions.

\section{Related Work}

\subsection{Relationships Between Intelligence and Creativity: Intelligence is a Subset of Creativity}

Intelligence can be regarded as a subset of creativity, which means that the creativity can be achieved using intelligence approaches according to creativity achievement theories. Several theories have been proposed to support this relationship.

One of the most important supportive theories for this relationship is the investment theory [1]. Investment theory states that creative individuals act as investors in the stock market, and they buy low and sell high in the process of generating ideas $[1,2]$. Sternberg and Lubart explained that the six elements are imperative to achieve creativity: intelligence, knowledge, thinking styles, personality, motivation, and the environment. Intelligence is essential in creativity elements [2].

Knowledge is the basis of intelligence. In cognition psychology, intelligence can be categorised by fluid intelligence and crystallised intelligence [3]. Knowledge is similar to crystallised intelligence [2,3]. Fluid intelligence can be defined as a capability of solving problems [3]. This kind of ability can be used to analyse problems, recognise patterns, and identify relationships. Fluid intelligence includes inductive reasoning and deductive reasoning [5]. Crystallised intelligence can be defined as a capability of using the knowledge, experiences, and skills that the individual learned previously. It is distinct from memory, but it is also based on long-term memory. An individual can reason something by using vocabulary and general knowledge $[5,6]$.

In the theory of Sternberg and Lubart, knowledge is a double-edged sword [4]. On one hand, people need to learn knowledge to understand the world. On the other hand, knowledge may impede creativity by leading the individual to be entrenched [3, 4]. To avoid entrenching one's thinking style, creative computing can help achieve multidisciplinary approaches by combining different subjects (this will be explained later) [4]. To pursue this creative thinking style, the individual should have a certain personality to defy crowd comments and be motivated to persist on encountering the obstacles in the process of pursuing creativity [4].

Another significant support theory is the triarchic theory by Sternberg [8]. The triarchic theory suggests that intelligence could achieve creativity, but three essential abilities were required: synthetic, analytical, and practical abilities [8].

The synthetic ability means considering the problem in a distinct way, which involves three knowledge acquisition components:

- selective encoding: to distinguish the related information from the unrelated information,

- selective combination: to combine related information in a creative way, and

- selective comparison: to connect new information to old information in a creative way [7].

Considering a problem is not only an ability, but also an attitude. This can be used to generate new ideas that guide in a completely different way and to assist the individual in solving a problem effectively [7, 8]. For example, Bohr's model related to the atom is a miniature version of the solar system, the result of applying selective comparison insights [8].

Another aspect of intelligence is the analytical part of intelligence, which is included in creativity. The analytical part is measured in part by conventional tests of intelligence. The value of each idea can be judged. The ideas that are worthy of pursuing can remain. When a valuable idea is found, the strengths and weaknesses of the ideas can be assessed using one's analytical abilities $[4,7,8]$.

The third aspect is practical ability, which can be applied to use intellectual skills in any context. As creative ideas may be difficult to be accepted, it is essential for individuals to learn how to communicate ideas effectively and persuade others to accept these ideas $[7,8]$. 
Based on Bloom's taxonomy of educational objectives, Smith's hierarchy theory is mainly about a cognitive process [9]. Taxonomy posited that the cognitive process can be a combination of the cumulative and hierarchical continuum [10]. The whole process starts with knowledge and proceeds with comprehension, application, analysis, synthesis, and evaluation. The first four elements are intellectual abilities and the last two are creative abilities, which require the potential skills in intelligence and generate creativity [9, 10]. To achieve creativity, there are three manners based on three theories: combinational theory, transformational theory, and exploratory theory. Boden stated that familiar ideas, such as poetic imagery and analogy, can be combined using the combinational manner [11]. The transformational manner and exploratory manner focus on conceptual spaces of the ideas. The transformational manner aims at reducing or expanding the conceptual space to create a new structure of the idea. The exploratory manner aims at changing the current structure of the idea to another one [11]. Three types of manners are used in the approach of this research to achieve creativity.

\subsection{Artificial Neural Network}

In this research, an ontology in the context of the semantic web can be generated using ANN. Ontology matching in the semantic web establishment requires a back-propagation neural network. Categories in an undefined dataset can be discovered through the clustering algorithm and the self-organising map (SOM) neural network.

Training for the weight and bias of the back-propagation formula is the kernel part and could influence further implementation. The process of training can be concluded in five steps:

- Collecting sample (Ai, Bi) from the sample dataset D;

- Calculating predicted results R from the BP algorithm;

- Calculating the difference between the predicted results and real results in the dataset, $\mathrm{B}=\mathrm{Bi}-\mathrm{R}$;

- Adjusting the weight value and bias value based on the difference value B; and

- Iterating steps 1-4 for each sample data item until the errors of predicting is within the threshold value $[12,13,14,15]$.

In the BP algorithm, the gradient decrescent finds the weight and bias value in the iteration. The gradient decrescent approach needs a cost function to operate more easily in the processing:

$$
C(w, b)=\left(\frac{1}{2 n}\right)\|y(x)-a\|^{2}
$$

where

a: represents the real results vector when inputting $\mathrm{x}$

w: represents the vector of weights

b: represents the vector of bias

$\mathrm{n}$ : represents the number of training data

$\mathrm{y}(\mathrm{x})$ : is the real output results vector $[12,13]$.

The formula aims to find the appropriate weight and bias to minimise the value of $\|y(x)-a\|$. This means making the prediction results approach the real results. Therefore, the difference between the real results and the output results can be confirmed using the cost function to minimise.

Based on the processing steps of the BP neural network algorithm, the pseudocode is:

Input $D$

Initialising $w, b$

For $x, y$ in $D$ :

Calculating the errors $\delta$

Calculating the random gradient value $\nabla \mathrm{C}$

Updating $w, b$ basing on the formula

End for

Until reach the threshold.

Output prediction accuracy, $w, b$. 


\subsection{Semantic Web}

Semantic web is a prospect for the future Internet. Many scholars agree to combine the semantic web with Web 3.0 as one of the characteristics of the new Internet era. Basically, the semantic web is an artificial Internet with functions on understanding concepts and words and comprehending logical relationships between them. Communication efficiency and values are improved by the semantic web [16, 17, 20]. The seven levels of the semantic web include the Unicode and URL, mark-up language, resource description framework (RDF), ontology vocabulary level, logic, proof, and trust [16, 17, 18, 20].

Axiom and principles will be provided at the logic level [20]. After the logic level has been established, resources, relationships between resources, and references results will be verified using logic inferences. The transfer happens along with a digital signature through the proof level. The output of semantic web reliability and satisfaction of users is evidenced by establishing relevant trust relationships [19].

Many modelling languages have been provided by the semantic web in their level of expressivity. Different information from different people can be expressed using tools that were constituted by modelling languages. RDF is the abbreviation for resource description framework. The rest of the semantic web is based on RDF. Basic statements from everyone can be collected by RDF and structured in the same model. Different models can be merged to form an integrated one [20]. OWL is the abbreviation for web ontology language. Constraints specifically between classes, entities, and properties can be described by Modellers. Expressivity of logic is brought to the semantic web by OWL [19]. Distributed data, a fundamental issue, is managed by RDF in the semantic web. The architecture of web and other proven and similar features are relied on by RDF to build a foundation for the distribution network [19, 20]. The RDF parser, serializer, stores, and query engines are parts of the system. Mata data, which is used for demonstrating other data, is also implemented in the semantic web [19, 20].

An example of RDF expression is:

\section{$<r d f: R D F$}

Xmlns:mfg=" http://www.londonstockexchange.com/statistics/companies-and-issuers/companyonlondonstockexcahnge\#" xmlns:rdf=" http://www.londonstockexchange.com\#">

<mfg:Company rdf:about=http://www.londonstockexchange.com/statistics/companies-andissuers/companyonlondonstockexchange\#lpmplc>

$<m f g:$ industry $>$ Financial $</ m f g:$ industry>

$<m f g:$ country $>U K</ m f g:$ country $>$

$<m f g$ :region > Europe </mfg:region>

$\langle$ mfg:marketCap >43.91</mfg:marketCap>

$</ m f g: c o m p a n y>$

$<m f g:$ Company

rdf:about=http://www.londonstockexchange.com/statistics/companies-andissuers/companyonlondonstockexchange\#GLANBIAplc>

$<m f g$ :industry>customerGoods </mfg:industry>

$<m f g:$ country $>$ Ireland $</ m f g:$ country $>$

$<m f g$ :region $>$ Europe $</ m f g:$ region $>$

$<$ mfg:marketCap >3638.24</mfg:marketCpa>

$\langle/ r d f: R D F>$

$</ m f g:$ company>

\subsection{Creative Computing}

Creative computing is now drawing attention because novel ideas for solving problems can be provided through creative computing methods [25]. As a general definition describes, creative computing refers to a computing activity that is novel, astonishing, and useful, and this computing can be applied to solve practical problems [21]. Each characteristic is intolerable [24]. Creativity is an ability to achieve creative ideas and artefacts [21].

The meaning of creative computing is with flexibility and probabilistic characteristics in the Internet area. More essentially, the hardware developed rapidly and constructed an excellent environment for software progression [22]. Creativity can be divided into H-creativity, P-creativity, exploratory creativity, transformational creativity, and combinational creativity [26]. H-creativity refers to historical creativity, which means this creative service or experiment has never been found before in the world. P-creativity is short for psychological creativity or personal creativity, which is at the personal level [27]. In addition, the exploratory creativity stands for an existing conceptual space conducting the research to achieve creativity [24]. 
Moreover, transformational creativity stands for creating a new conceptual space based on transforming an existing conceptual space. The combinational creativity aims at combining similar ideas and thoughts to establish new methods [24]. Integrating related former research in distinct domains is the core purpose of creative computing [26]. Creativity cannot be considered as a sort of algorithm but rather as a form of navigation that has the capability to guide researchers to be creative [23].

\section{Solution Generation Approach based on Intelligence Being a Subset of Creativity (SGIC)}

The proposed approach is a Solution Generation approach based on intelligence being the subset of creativity theory (SGIC). Three kernel steps are query analysis, information explanation, and creative solution achievement.

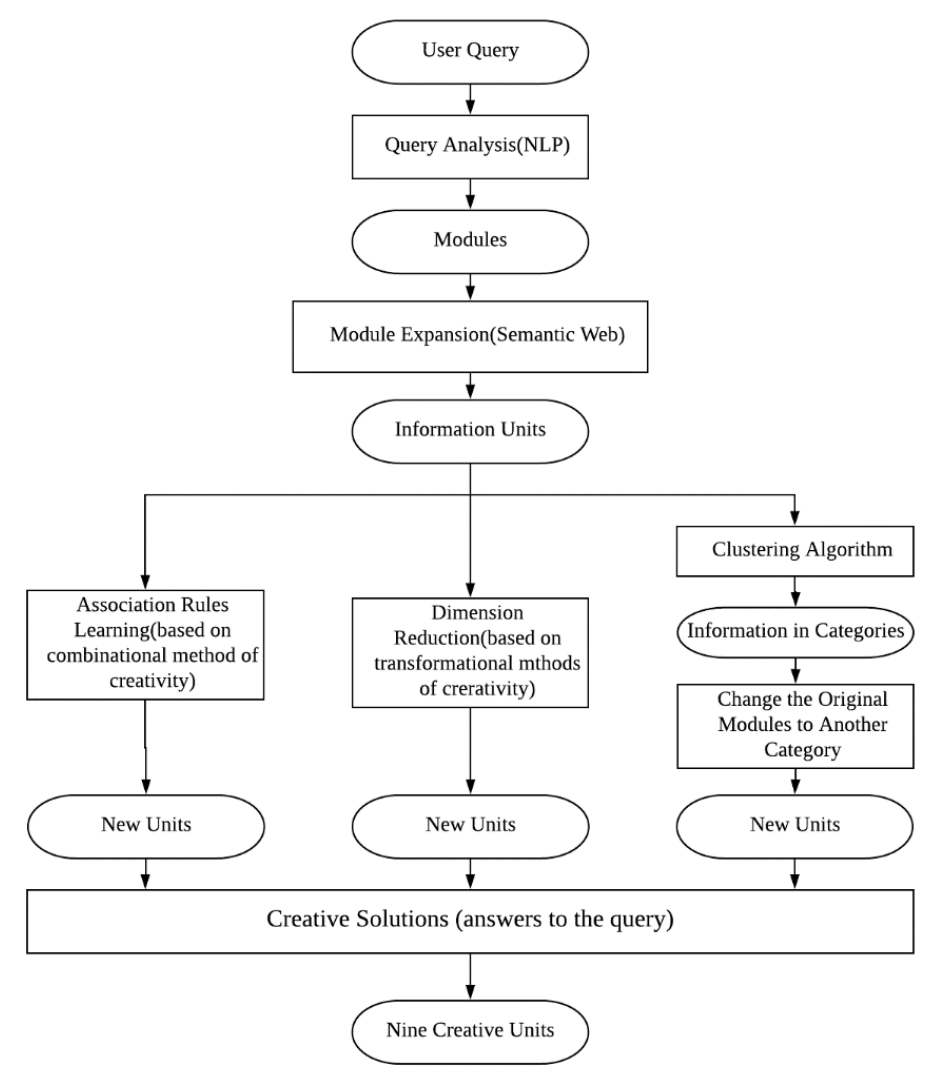

Figure 1. Solution generation based on intelligence being subset of creativity theory approach

\subsection{Query Analysis}

The user query is usually a sentence and includes kernel information. To make the computer process easier, the query is necessary to be divided into pieces. The natural language process can divide the sentence into subjective word, predicate word, objective word, and other qualifiers such as basic modules. The kernel words can represent the requirements from the user and can influence solution discovery through further processing. This step is processed by NLTK (natural language toolkit in Python) [28].

\subsection{Information Expansion}

Before the semantic expansion starts, the ontology matching should be done by using the BP neural network. The axioms, concepts, relationships, and relevant instances can be connected to an alignment by general matching in the semantic web. This kind of work is often completed manually. In the matching process, distinct semantic aspects, involving the name of the concept, property of the concept, and relationships between concepts, have different contributions to the matching outcomes. Thus, the matching process can be made effectively, and matching results can be accurate by generating a suitable weight for each aspect. The weight and bias in the BPNN should be pre-trained with data in same subject of the user's query. 
The formula between concept and each related element is:

$$
\mathrm{C}=A_{1} x_{1}+A_{2} x_{2}+A_{3} x_{3}+\cdots+A_{i} x_{i}
$$

$\mathrm{C}$ represents the concept;

i represents the number of the elements;

$x_{i}$ represents each element;

$A_{i}$ represents weights.

To obtain the weights of the formula, the information in the unit should be transferred to vectors, regarding the kernel concept as the unit matrix. The training formula of BPNN is:

$$
\mathrm{C}(\mathrm{A}, \mathrm{b})=\left(\frac{1}{2 \mathrm{i}}\right)\|\sigma(\mathrm{x})-\mathrm{a}\|^{2}
$$

$\sigma(\mathrm{x})$ represents each appropriate matching with the concept; a represents the appropriate vectors when $x_{i}$ is input. the bias.

The training stops when the result $\mathrm{C}(\mathrm{A}, \mathrm{b})$ is convergent. Value $\mathrm{A}$ is the weight between the relevant element, and $\mathrm{b}$ is

\subsection{Creative Solution Generation}

In this step, the expanded information units are processed in three types of approaches, combinational, transformational, and exploratory approach, which are based on Boden's creativity achievement theory [11].

The combinational-theory-based approach aims at combining similar units between different sets.

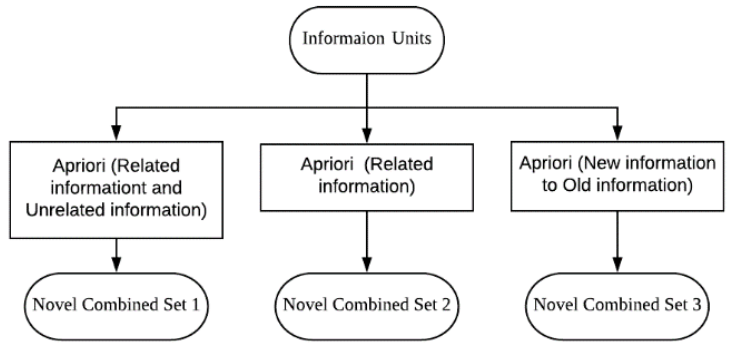

Figure 2. Combinational creativity achievement approach

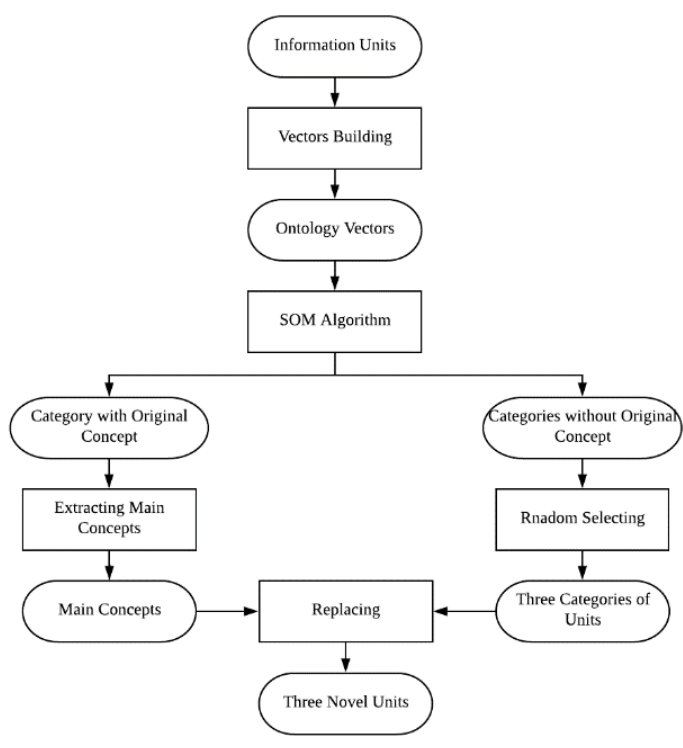

Figure 3. Exploratory-method-based approach

The association rules learning algorithm can be used to achieve this approach (Fig. 2). The Apriori algorithm is a seminal algorithm in the association rules learning subject [31]. The itemset that is above the minimum support can be called the frequent itemset. If the itemset support is less than the minimum support value, the relevant superset is not a frequent itemset, but a downward closure property.

The Apriori algorithm makes several passes for the data [29, 30]. Initially, the frequent itemsets is determined by counting the support of the individual items. Secondly, previous pass is used for building the new potentially frequent itemset (candidate itemset). The support is counted during the pass over the data. Eventually, frequent itemsets are collected and are sent for the next pass. This process can be defined as an iteration, which will be stopped until no more frequent itemset can be found [29, 32]. 
It is essential to complete the association rules detection in advance and save them in a database for further use. Initially, the Apriori algorithm should train the datasets of business behaviours and relevant changing data to find relationships between them. The process of the Apriori algorithm is:

$D=\{$ frequent 1-itemsets $\}$;

for $\left(k=2 ; F_{k-1}=\varnothing ; k++\right)$ do begin

$C_{k}=$ Apriori-gen $\left(D_{k-1}\right)$;

For each transaction $t \in D$ do begin

$C_{t}=\operatorname{subset}\left(C_{k}, t\right)$;

For each candidate $c \in C_{t}$ do

c.count ++ ;

end

$D_{k}=\left\{c \in C_{k} \mid\right.$ c.count $\geqslant$ minsup $\} ;$

end

Answer $=\cup_{k} D_{k}$;

The data in the dataset $\mathrm{D}$ can be scanned to count the appearance numbers (support number). Then, the data is ranked from high support to low support and can be selected by the minimum support number that the user determined.

Additionally, the algorithm can detect a data combination, which contains two or three concepts in one subset, and the same process can complete the support counting and ranking. The three passes in the Apriori algorithm can achieve the three aspects of combinations from the triarchic theory. The output of this approach can be three combined sets including problem solutions.

The transformational-theory-based approach aims at achieving creative outcomes by reducing the conceptual space dimensions. New structures can be generated, and "surprising" and "novel" ideas may be achieved. Through the dimension reduction algorithm, principle components analysis can be used to achieve this approach.

The input data is the output of semantic web, expanded information units. Based on the weight of each ontology that BPNN calculated in step two, all the information can be transferred into vectors (regarding the basic concept as a unit matrix).

The PCA algorithm can be achieved by using mean value, covariance of the matrix, and the normalised data. To finish obtaining the principle components, the mean value of each subject of data initially can be calculated using the PCA algorithm for further use. Then, the relevant feature value and feature vectors can be calculated by PCA. The new feature values are sorted in a numeric order. Top three features values can be selected, and related features vectors can be used to establish a new space in this step. The normalised data is delivered to the new space established by the feature vectors. The core code to achieve PCA algorithm is presented:

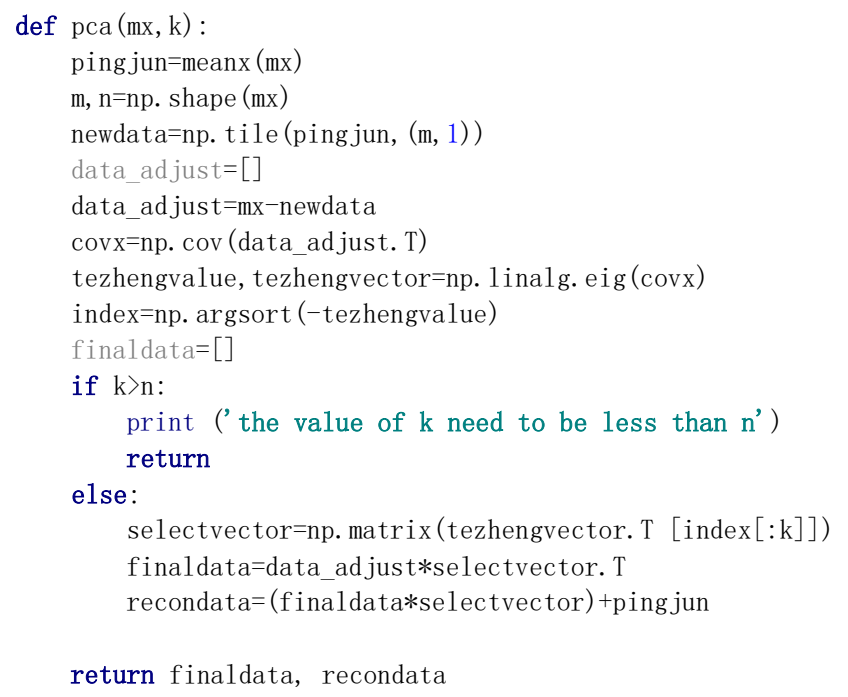

Based on the PCA, the conceptual space dimensions can be reduced, and new information units can eventually be achieved. The final output of this step can be three units (principle components), and the others are dropped. 
The exploratory-method-based approach aims at changing the conceptual space, which means changing the dimensions of the target concept. To achieve this, the information units should be divided into categories in an unsupervised manner. Then, the target concept is transferred to another category to make the structure of the conceptual space being changed (Fig. 3).

The input of this step is the expanded information units. The information is required to be transferred to vectors based on the basic concept unit matrix, which is the same as the processing in the transformational approach.

The SOM algorithm can achieve this approach. The general SOM algorithm is a kind of unsupervised learning to cluster the data. Basic steps of the SOM algorithm can be explained as:

- $\quad$ Randomise the node weight vectors in a map;

- Randomly pick an input vector; the input set has D dimensions. The input vector is

$$
\mathrm{x}=\left\{x_{i} \mid i \in 1,2 \ldots, D\right\}
$$

Use the Euclidean distance formula (2) to discover the similarity between the input vector and the map node weight vector.

Find the node that the distance $\mathrm{d}$ is the smallest, which can be defined as the best matching unit (BMU):

$$
d_{j}(\mathrm{x})=\sum_{i=1}^{D}\left(x_{i}-w_{j i}\right)^{2}
$$

- Updating the weight vector of the nodes $\mathrm{T}$ in the neighbourhood of the BMU and the BMU itself. They should be nearer to the input vector;

$$
T_{j, I(x)}=\exp \left(-\frac{s_{j, I(x)}^{2}}{2 \partial^{2}}\right)
$$

- $\quad$ Update the parameter of the node by using the gradient decrescent

- $\quad$ Iteration until the $\triangle w_{j i}$ is convergent.

$$
\triangle w_{j i}=\eta(t) * T_{j, I(x)}(t) *\left(x_{i}-w_{j i}\right)
$$

After the parameters of the SOM algorithm are completed, the clustering for the information-unit-based vectors can be completed. The final output is a series of categories. The category that contains the answers to the original user's query concept should be found. Then, three other categories are randomly selected, which can output three new units, involving the underlying creative solutions for the query. The final outputs of this step are nine creative units including solutions for the query.

\section{Experiments and Results}

To explain the whole SGIC process explicitly, a case on the investment portfolio is presented in this section. The simulative user query is about an investment portfolio decision of "How to generate a stable benefit investment portfolio in June 2018 within a range of fluctuation of 1\%"? After the query is divided and analysed by NLP, "stable benefit", "investment portfolio", "June 2018", and "fluctuation if 1\%" are output by SGIC. The information can be expanded through the semantic web technique with a weighted ontology (built by BPNN). The information is obtained from the industries, companies, and markets that the companies are in. Information of the top 100 companies was selected in this case.

The expanded information is processed by three sub-approaches in step three. In the combination part, the information of selected information units are:

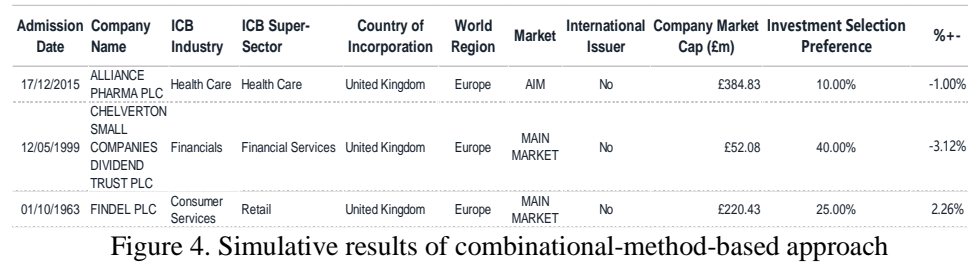

In the transformation part, the information is transferred to vectors based on the weighted ontology system. The main principles in the information units can be discovered by PCA. A general diagram of the new space and the distributions situation of the principle components can be presented in Fig. 5. The main components can be extracted. Three of the outcomes are selected as the output of this part. New units are achieved for the user about the investment.

In the exploratory part, the information units are required to be transferred to vectors. An unsupervised learning clustering can be completed by dealing with vectors through the SOM algorithm, achieving categories in Fig. 6. After the normalisation, the data categories can be presented in Fig. 7. Then, the main query concept can be moved to the other three randomly selected categories to replace the similar components. Three novel units are achieved in this manner. 
The final output will be nine information units that include the basic concept information and relevant solutions for the query. The users can obtain the potential investment portfolio with companies' information and suggestions from the nine outcomes.

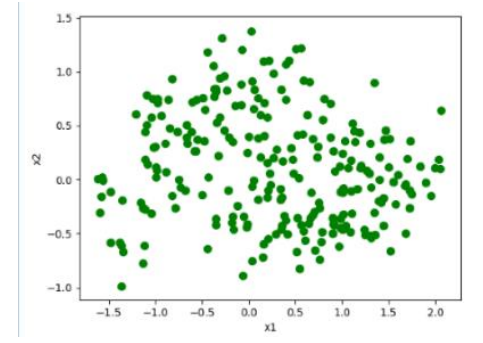

Figure 5. Simulative results of PCA

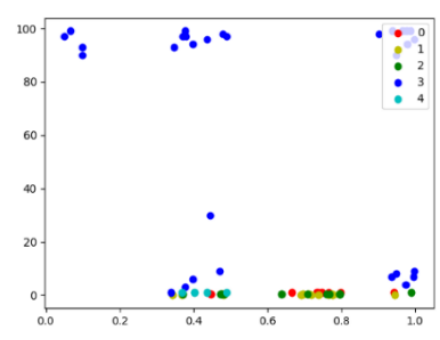

Figure 6. Simulative results of SOM algorithm

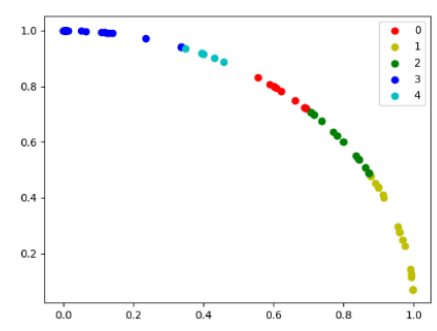

Figure 7. Normalised simulative results of SOM

\section{Conclusions}

A solution generation system, SGIC, established on the basis that intelligence is the subset of the creativity was proposed. The algorithms were developed according to creativity theories to achieve solution generation, where creativity was simulated through combinational, transformational, and exploratory manners, which can all be generated through artificial intelligence algorithms. To make the process explicit and logical, a detailed creative computing method was illustrated for interdisciplinary problem solving. To summarise, the SGIC system is implemented through combining intelligence algorithms (including BPNN, SOM, and PCA), Apriori algorithm, Semantic Web, and creativity theories (investment model, triarchic theory, and Boden's three creativity theories). Nine creative solutions were generated for the user's query as a result.

Future work can also be suggested. First, the generated outputs/solutions are now in nine units, mixed with other information. The solution extraction is therefore needed. Second, these nine units may include several solutions. To provide the most appropriate solution to the user, a more comprehensive ranking algorithm is to be developed.

\section{References}

1. R. Sternberg and T. Lubart, Defying the Crowd: Cultivating Creativity in a Culture of Conformity, Free Press, American Psychological Association, New York, USA, 1995.

2. R. Sternberg, Successful Intelligence, First Agency Publishing, American Psychological Association, New York, USA, 1997.

3. R. Sternberg, Beyond IQ: A Triarchic Theory of Human Intelligence, Cambridge University Press, Press Syndicate of University of Cambridge, Cambridge, UK, 1985.

4. $\quad$ R. Sternberg, The Triarchic Mind: A New Theory of Human Intelligence, Viking Press, New York, USA, 1988.

5. R. Cattell, "Theory of Fluid and Crystallized Intelligence: A Critical Experiment", Journal of Educational Psychology, American Psychological Association, New York, USA, vol. 54, no. 1, pp. 1-8, 1963.

6. R. Cattell, "Theory of Fluid and Crystallized Intelligence: A Critical Experiment", Journal of Educational Psychology, American Psychological Association, New York, USA, vol. 54, no. 1, 1963.

7. R. Sternberg, and T. Lubart, "Investing in Creativity", American Psychologist, American Psychological Association, New York, USA, vol. 51, no. 7, p. 677, 1996.

8. R. Sternberg, "Implicit Theories of Intelligence, Creativity, and Wisdom", Journal of Personality and Social Psychology, American Psychological Association, Washington, USA, vol. 49, no. 3, p. 607, 1985.

9. I. Smith, "IQ, Creativity, and the Taxonomy of Educational Objectives: Cognitive Domain", Journal of Experimental Education, Taylor and Francis Co., London, UK, vol. 38, no. 4, pp.58-60, 1970.

10. I. Smith, "IQ, Creativity, and Achievement: Interaction and Threshold", Multivariate Behavioural Research, American Psychological Association, New York, USA, vol. 6, no. 1, pp. 51-62, 1971.

11. M. Boden, "Creativity and Artificial Intelligence", Artificial Intelligence, Elsevier, Netherland, vol. 103, no. 1-2, pp. 347-356, 1998.

12. P. Heermann and K. Nahid, "Classification of Multispectral Remote Sensing Data Using a Back-Propagation Neural Network", IEEE Transactions on Geoscience and Remote Sensing, London, UK, vol. 30, no. 1, pp. 81-88, 1992.

13. T. Lee, "Back-Propagation Neural Network for Long-Term Tidal Predictions", Ocean Engineering, Elsevier, New York, USA, vol. 31, no. 2, pp. 225-238, 2004.

14. S. Haykin, “A Comprehensive Foundation”, IEEE Neural Networks, London, UK, vol. 2, no., p. 41, 2004.

15. M. Hagan, B. Howard and H. Mark, Neural Network Design, Boston PWS Publishing, Boston, USA, vol. $20,1996$.

16. T. Berners-Lee, H. James and L. Ora, "The Semantic Web", Scientific American, Natural American Inc., USA, vol. 284, no. 5, pp. 34-43, 2001.

17. S. Nigel, T. Berners-Lee and W. Hall, "The Semantic Web Revisited", IEEE Intelligent Systems, New York, USA, vol. 21, no. 3, pp. 96-101, 2006. 
18. S. Bechhofer, "OWL: Web Ontology Language", Encyclopedia of Database Systems, Springer, New York, USA, pp. 2008-2009, 2009.

19. A, Bernstein, H. James, and N. Natalya, "A New Look at the Semantic Web", Communications of the ACM, New York, USA, vol. 59, no. 9, pp. 35-37, 2016.

20. D. Allemang and H. James, Semantic Web for the Working Ontologist: Effective Modelling in RDFS and OWL, Elsevier, New York, USA, 2011.

21. L. Zhang, and H. Yang, "Definition, Research Scope and Challenges of Creative Computing", 19th IEEE International Conference on Automation and Computing (ICAC), London, UK, pp. 1-6, 2013.

22. A. Hugill and H. Yang, "The Creative Turn: New Challenges for Computing", International Journal of Creative Computing (IJCrC), Inderscience Enterprises Ltd., Olney, UK, vol. 1, no. 1, pp. 4-19, 2013.

23. W. Schoenmakers and G. Duysters, "The Technological Origins of Radical Inventions", Research Policy, Elsevier, The Netherlands, vol. 39, pp. 1051-1059, 2010.

24. H. Yang, D. Jing, and L. Zhang, "Creative Computing: An Approach to Knowledge Combination for Creativity?", IEEE Symposium on Service-Oriented System Engineering (SOSE), London, UK, pp. 407-414, 2016.

25. L. Zou, Q. Liu, C. Zhang and H. Yang, "An Approach to Applying Creative Computing in Tourism by Constructing a Big Data based Knowledge System Framework", 22nd IEEE International Conference on Automation and Computing (ICAC), London, UK, pp. 244249, 2016.

26. D. Jing, and H. Yang, "Creative Computing for Bespoke Ideation", $39^{\text {th }}$ IEEE Computer Software and Applications Conference (COMPSAC), Taichung, Taiwan, China, vol. 1, pp. 34-43, 2015.

27. L. Zhang and H. Yang, "Knowledge Discovery in Creative Computing for Creative Tasks", Creativity in Intelligent Technologies and Data Science: First Conference(CIT\&DS), Springer, London, UK, vol. 535, p. 93, 2015.

28. J. Perkins, Python 3 Text Processing with NLTK 3 Cookbook, Packt Publishing Ltd, Birmingham, UK, 2014.

29. X. Wu, et al, "Top 10 Algorithms in Data Mining", Knowledge and Information Systems, Springer, London, UK, vol. 14, no. 1, pp. 137, 2008

30. M. Al-Maolegi and A. Bassam, "An Improved Apriori Algorithm for Association Rules", ArXiv.org., Cornell University Library, USA, 2014.

31. J. Dongre, L. Gend and S. V. Tokekar, "The Role of Apriori Algorithm for Finding the Association Rules in Data Mining", IEEE International Conference on Issues and Challenges in Intelligent Computing Techniques (ICICT), India, 2014.

32. W. Schoenmakers, and G. Duysters, "The Technological Origins of Radical Inventions", Research Policy, Elsevier, The Netherlands, vol. 39, pp. 1051-1059, 2010.

Qinyun Liu received his BSc degree in International Economics and Trade from Hebei University of Technology, China, in 2013 and his MSc degree in International Business from Robert Gordon University, UK in 2015. Currently, he is a PhD candidate in Creative Computing at Bath Spa University. His research interests include creative computing, particularly the aspect of linking computer science with business.

Professor Hua Zhou received his BSc degree in Computational Mathematics from Yunnan University, China in 1984, his MSc degree in Computer Science from Jilin University, China in 1990, and his PhD degree in Software Engineering from DE Montfort University, UK in 2004. He was a faculty member at two universities, i.e., Yunnan University, China from 1984 and Southwest Forestry University, China from 2017. He has published over 60 refereed journals and conference papers. His research interests include software engineering, artificial intelligence, data science, big data technology, and web and distributed computing.

Professor Hongji Yang received his BSc and MSc degrees in Computer Science from Jilin University, China in 1982 and 1985, respectively, and his PhD degree in Computer Science from Durham University, UK in 1994. He was a faculty member at several universities, i.e., Jilin University, China from 1985, Durham University, UK from 1989, De Montfort University, UK from 1993, Bath Spa University, UK from 2013, and Leicester University, UK from 2018. He has published over 400 refereed journal and conference papers. He became an IEEE Computer Society Golden Core Member in 2010. He has also been a member of the Engineering and Physical Sciences Research Council (EPSRC), Peer Review College since 2003. He is the Editor-in-chief of the International Journal of Creative Computing, InderScience. His research interests include software engineering, creative computing, and web and distributed computing.

William Cheng-Chung Chu is a distinguished professor of the Department of Computer Science and the Director of Software Engineering and Technologies Centre of Tunghai University. He has served as the Dean of Research and Development Office at Tunghai University from 2004 to 2007, Taiwan. From 1994 to 1998, he was the dean of the Engineering College. He is serving as the associate editor for IEEE Transaction on Reliability (TRel), International Journal of Advancements in Computing Technology (IJACT). He has served as an associate editor for the Journal of Software Maintenance and Evolution (JSME) and the Journal of Systems and Software (JSS). His current research interests include software engineering, cloud computing, and data analytics. Dr. Chu received his MS and PhD degrees from Northwestern University in Evanston Illinois, both in Computer Science. He has edited several books and published over 200 refereed papers and book chapters as well as participated in many international activities, including organizing international conferences, serving on the steering committee for COMPSAC and APSEC as well as on the program committee for more than 70 international conferences. 